\title{
A PARTITION THEOREM FOR $[0,1]$
}

\author{
H. J. PRÖMEL AND B. VOIGT
}

(Communicated by Andreas R. Blass)

ABSTRACT. We prove a Hindman-type partition theorem for Baire partitions of $[0,1]$.

\section{INTRODUCTION AND STATEMENT OF RESULTS}

Hindman's theorem on finite sums [Hin] asserts that for every partition of the positive integers into finitely many classes there exist infinitely many positive integers such that all their finite sums (without repetition) belong to the same class.

In this note we investigate partitions of positive reals less than 1 into finitely many classes and ask whether there exist infinitely many reals with all their sums (finite or infinite, but without repetition) belonging to the same class. Of course, for an infinite sum this necessarily requires convergence. It is well known that the axiom of choice implies the answer to be no:

Theorem. Assuming the axiom of choice, there exists a partition $[0,1]=\mathscr{A}_{0} \cup \mathscr{A}_{1}$ such that for every sequence $\left(t_{i}\right)_{i \in \mathbf{N}}$ of positive reals satisfying $\sum_{i \in \mathbf{N}} t_{i}<1$ there exist infinite subsets $I, J \subseteq \mathbf{N}$ such that $\sum_{i \in I} t_{i} \in \mathscr{A}_{0}$ and $\sum_{i \in J} t_{i} \in \mathscr{A}_{1}$.

Proof. A standard diagonalization argument, based on the fact that there exist continuum many sequences $\left(t_{i}\right)_{i \in \mathbf{N}}$ with $\sum_{i \in \mathbf{N}} t_{i}<1$ and each such sequence admits continuum many distinct sums $\sum_{i \in I} t_{i}, I \subseteq \mathbf{N}$.

Here we show that if Zermelo-Fraenkel set theory is consistent, so is consistent Zermelo-Fraenkel set theory plus "the answer to the above question is YES."

As a matter of fact, we prove a somewhat more informative statement which in particular shows that the answer is always YES, provided the partition has been defined constructively.

Theorem. Let $[0,1]=\mathscr{A}_{0} \cup \cdots \cup \mathscr{A}_{r-1}$ be a partition of $[0,1]$ into sets having the property of Baire. Then there exists a sequence $\left(t_{i}\right)_{i \in \mathrm{N}}$ of positive reals with

Received by the editors December 23, 1988 and, in revised form, July 27, 1989.

1980 Mathematics Subject Classification (1985 Revision). Primary 05A17, 26A21. 03E20.

Key words and phrases. Ramsey theorems, finite and infinite sums, Baire sets. 
$\sum_{i \in \mathbf{N}} t_{i}<1$ and there exists $j<r$ such that $\sum_{i \in I} t_{i} \in \mathscr{A}_{j}$ for all nonempty subsets $I \subseteq \mathbf{N}$.

This is a Hindman-type partition theorem for Baire partitions of $[0,1]$; compare [GRS].

As the referee pointed out to us, with respect to partitions into Borel sets the result can be deduced from the result of Galvin and Prikry that Borel sets are Ramsey [GP]. In fact, let $\sum_{i=1}^{\infty} s_{i}$ be any convergent series of positive numbers. Since the mapping $I \rightarrow \sum_{i \in I} s_{i}$ is continuous, a Borel partition $\mathbf{R}=\mathscr{A}_{0} \cup \cdots \cup$ $\mathscr{A}_{r-1}$ induces a Borel partition of $\mathscr{P}(\mathbf{N})$. By the Galvin-Prikry theorem, for some $j \in\{0, \ldots, r-1\}$ there is an infinite set $K \subseteq \mathbf{N}$ such that $\sum_{i \in I} s_{i} \in \mathscr{A}_{j}$ for every infinite set $I \subseteq K$. Now let $\left(K_{i}\right)_{i \in \mathbf{N}}$ be a sequence of disjoint infinite subsets of $K$, and let $t_{i}=\sum_{k \in K_{i}} s_{k}$; then $\sum_{i \in I} t_{i} \in \mathscr{A}_{j}$ for every nonempty set $I \subseteq \mathbf{N}$. The Galvin-Prikry theorem has been conceptually generalized by dual Ramsey theorem of Carlson and Simpson [CS]. The Carlson-Simpson result has been strengthened by the authors [PV]; however, also this strengthened version does not imply directly the theorem of this paper.

Shelah [She] has shown that "Consistency of $Z F$ " implies "Consistency of $Z F$ plus the axiom of dependent choices plus every set of reals has the property of Baire." Hence, the theorem admits the following corollary:

Corollary. If Zermelo-Fraenkel set theory is consistent, so is consistent ZermeloFraenkel set theory plus the axiom of dependent choices plus "for every partition $[0,1]=\mathscr{A}_{0} \cup \cdots \cup \mathscr{A}_{r-1}$, where $r \in \mathbf{N}$, there exists a sequence $\left(t_{i}\right)_{i \in \mathbf{N}}$ and there exists $j<r$ such that $\sum_{i \in I} t_{i} \in \mathscr{A}_{j}$ for all nonempty $I \subseteq \mathbf{N}$."

With the slightly stronger hypothesis that $Z F$ plus "there exists an inaccessible cardinal" is consistent the corollary follows also from the result of Mathias [Math] that all subsets of $\mathscr{P}(\mathbf{N})$ are Ramsey in Solovay's model [Sol].

\section{Proof of THEOREM}

Notation. For $a \in[0,1]$ and $\varepsilon>0$ we denote by $\mathscr{B}(a, \varepsilon):=\{b \in \mathbf{R}|| a-b \mid<$ $\varepsilon\}$ the open ball of radius $\varepsilon$ with center $a$.

Lemma A. Let $N$ be a positive integer, let $a_{0}, \ldots, a_{N-1}$ be positive reals, let $b \in[0,1]$, let $\varepsilon>0$ and let $\mathscr{D} \subseteq \mathbf{R}$ be dense open.

Then there exist $\delta>0$ and a positive real $c \in \mathscr{B}(b, \varepsilon)$ such that

$$
\begin{gathered}
\mathscr{B}(c, \delta) \subseteq \mathscr{B}(b, \varepsilon), \\
\mathscr{B}\left(a_{i}+c, \delta\right) \subseteq \mathscr{B}\left(a_{i}+b, \varepsilon\right) \cap \mathscr{D} \text { for every } 0 \leq i<N .
\end{gathered}
$$

Proof. An obvious induction on $N$ :

Assume that $c^{\prime}$ and $\delta^{\prime}$. have been constructed with respect to $a_{0}, \ldots, a_{N-2}$. As $\mathscr{D}$ is dense open, the intersection $\mathscr{D} \cap \mathscr{B}\left(a_{N-1}+c^{\prime}, \delta^{\prime}\right)$ contains an open interval. Hence there exist $\delta>0$ and $c \in \mathscr{B}\left(c^{\prime}, \delta^{\prime}\right) \subseteq \mathscr{B}(b, \varepsilon)$ such that (2) is satisfied for $i=N-1$. 
We can assume that $\mathscr{B}(c, \delta) \subseteq \mathscr{B}\left(c^{\prime}, \delta^{\prime}\right)$ and thus (2) is still satisfied for $i=0, \ldots, N-2$.

Lemma B. Let $\mathscr{A}$ be a set of positive reals having the property of Baire. Assume that $B(0, \varepsilon) \cap \mathscr{A}$ is nonmeager for every $\varepsilon>0$. Then there exists a sequence of positive reals such that $\sum_{i \in \mathrm{N}} t_{i}<1$ and such that $\sum_{i \in I} t_{i} \in \mathscr{A}$ for every nonempty $I \subseteq \mathbf{N}$.

Proof. As $\mathscr{A}$ has the property of Baire, it can be written as $\mathscr{A}=(\mathscr{O} \backslash \mathscr{M}) \cup$ $(\mathscr{M} \backslash \mathscr{O})$ for some open set $\mathscr{O}$ and some meager set $\mathscr{M}$. Let $\mathscr{D}_{i}$ be dense open sets such that $\mathscr{M} \subseteq \mathbf{R} \backslash \bigcap_{i \in \mathbf{N}} \mathscr{D}_{i}$.

Without loss of generality we can assume that $\mathscr{D}_{i+1} \subset \mathscr{D}_{i}$. Note that $\mathscr{B}(0, \varepsilon)$ $\cap \mathscr{O} \neq \varnothing$ for every $\varepsilon>0$. Let $t_{0}^{0}<\frac{1}{2}$ and let $0<\varepsilon_{0}<\frac{1}{2}$ be such that $\mathscr{B}\left(t_{0}^{0}, \varepsilon_{0}\right) \subseteq \mathscr{O}$.

By induction on $n$ there exist positive reals $t_{i}^{n}, 0 \leq i \leq n$ and there exists $\varepsilon_{n}>0$ such that

$$
\mathscr{B}\left(\sum_{i \in I} t_{i}^{n}, \varepsilon_{n}\right) \subseteq \mathscr{O} \cap \mathscr{D}_{n}
$$

for every nonempty $I \subseteq\{0, \ldots, n\}$. We perform the inductive step: Consider the ball $\mathscr{B}\left(0, \varepsilon_{n}\right)$. As $\mathscr{O} \cap \mathscr{B}\left(0, \varepsilon_{n}\right) \neq \varnothing$ there exist $t$ and $\varepsilon$ such that $\mathscr{B}(t, \varepsilon) \subseteq \mathscr{O} \cap \mathscr{B}\left(0, \varepsilon_{n}\right)$.

By Lemma A there exists $t_{n+1}^{n+1} \in \mathscr{B}(t, \varepsilon)$ and there exists $\delta_{0}>0$ such that $\mathscr{B}\left(t_{n+1}^{n+1}, \delta_{0}\right) \subseteq \mathscr{B}(t, \varepsilon)$ and such that

$$
\mathscr{B}\left(\sum_{i \in I} t_{i}^{n}+t_{n+1}^{n+1}, \delta_{0}\right) \subseteq \mathscr{D}_{n+1} \cap \mathscr{B}\left(\sum_{i \in I} t_{i}^{n}+t, \varepsilon\right)
$$

for all $I \subseteq\{0, \ldots, n\}$, where $\sum_{i \in \varnothing} t_{i}^{n}=0$ by definition.

By induction on $j$, using Lemma A, there exist $t_{n-j}^{n+1}$ and $\delta_{j+1}>0$ for $j=0, \ldots, n$ such that $\mathscr{B}\left(t_{n-j}^{n+1}, \delta_{j+1}\right) \subseteq \mathscr{B}\left(t_{n-j}^{n}, \delta_{j}\right)$ and such that

$$
\mathscr{B}\left(\sum_{i \in I} t_{i}^{n}+t_{n-j}^{n+1}, \delta_{j+1}\right) \subseteq \mathscr{D}_{n+1} \cap \mathscr{B}\left(\sum_{i \in I} t_{i}^{n}+t_{n-j}^{n}, \delta_{j}\right)
$$

for all $I \subseteq\{0, \ldots, n-j-1\}$.

Put $\varepsilon_{n+1}=\frac{1}{2} \cdot \delta_{n+1}$.

This completes the definition of the numbers $t_{i}^{j}, 0 \leq i \leq j$. Note that for fixed $i$ the sequence $\left(t_{i}^{j}\right)_{i \leq j}$ is a Cauchy sequence. We define $t_{i}=\lim _{j \rightarrow \infty} t_{i}^{j}$.

Now let $I \subseteq \mathbf{N}$ be nonempty. The crucial property of the construction is that

$$
\sum_{i \in I} t_{i} \in \mathscr{B}\left(\sum_{\substack{i \in I \\ i \leq m}} t_{i}^{m}, \varepsilon_{m}\right)
$$


for every $m \in \mathbf{N}$. In particular, by choice of $t_{m}^{m}$, this implies that

$$
\sum_{i \in I} t_{i} \in \mathscr{B}\left(t_{\min I}^{\min I}, \varepsilon_{\min I}\right) \subseteq \mathscr{O}
$$

Also,

$$
\sum_{i \in I} t_{i} \in B\left(\sum_{\substack{i \in I \\ i \leq n}} t_{i}^{n}, \varepsilon_{n}\right) \subseteq \mathscr{D}_{n}
$$

for every $n \in \mathbf{N}$. In other words,

$$
\sum_{i \in I} t_{i} \in \mathscr{O} \backslash \mathscr{M} \subseteq \mathscr{A}
$$

which completes the proof of Lemma B.

Proof of theorem. Let $[0,1]=\mathscr{A}_{0} \cup \cdots \cup \mathscr{A}_{r-1}$ be a partition into sets having the property of Baire. For every $\varepsilon>0$ some $\mathscr{B}(0, \varepsilon) \cap \mathscr{A}_{j}$ is nonmeager. Hence there exists $j<r$ such that $\mathscr{A}_{j}$ satisfies the hypothesis of Lemma B.

\section{Concluding REMARKS}

In view of Lemma $B$ the following result can be established:

Theorem. For every partition $[0,1]=\bigcup_{i \in \mathbf{N}} \mathscr{A}_{i}$ into countably many sets with the property of Baire there exists a sequence $\left(t_{i}\right)_{i \in \mathbf{N}}$ with $\sum_{i \in \mathbf{N}} t_{i}<1$ such that either

- there exists $j \in \mathbf{N}$ such that

$$
\sum_{i \in I} t_{i} \in \mathscr{A}_{j} \quad \text { for all nonempty } I \subseteq \mathbf{N},
$$

or

- for all nonempty $I, J \subseteq \mathbf{N}$ :

there exists $j \in \mathbf{N}$ with $\sum_{i \in I} t_{i} \in \mathscr{A}_{j}$ and $\sum_{i \in J} t_{i} \in \mathscr{A}_{j}$ if and only if $\min I=$ $\min J$.

More precisely, using the method of proof for Lemma B one can show Lemma C.

Lemma C. Let $\left(B_{n}\right)_{n \in \mathbf{N}}$ be a sequence of subsets of $\mathbf{R}$, each having the property of Baire. Suppose that, for each $\varepsilon>0$, there are infinitely many $n \in \mathbf{N}$ such that $B_{n} \cap(0, \varepsilon)$ is nonmeager. Then there exist a strictly increasing function $r: \mathbf{N} \rightarrow \mathbf{N}$ and a sequence $\left(t_{i}\right)_{i \in \mathbf{N}}$ of positive real numbers with $\sum_{i=1}^{\infty} t_{i}<1$, such that $\sum_{i \in I} t_{i} \in B_{r(\min I)}$ for every nonempty set $I \subseteq \mathbf{N}$.

This is a kind of canonizing theorem. With respect to Borel partitions a stronger result has been achieved in [PSV]. 
Problem. What are canonizing patterns for Baire mappings $\Delta:[0,1] \rightarrow \mathscr{X}$ into metric spaces $\mathscr{X}$ ?

\section{ACKNOWLEDGMENT}

We thank the referee, whose valuable remarks substantially improved the presentation of the paper.

\section{REFERENCES}

[CS] T. J. Carlson and S. G. Simpson, A dual form of Ramsey's theorem, Adv. in Math. 53 (1984), 265-290.

[GP] F. Galvin and K. Prikry, Borel sets and Ramsey's theorem, J. Symbolic Logic 38 (1973), 193-198.

[GRS] R. L. Graham, B. L. Rothschild, and J. Spencer, Ramsey theory, Wiley, New York, 1980.

[Hin] N. Hindman, Finite sums from sequences within cells of a partition of $N, \mathrm{~J}$. Combin. Theory Ser. A 17 (1974), 1-11.

[Math] A. R. D. Mathias, Happy families, Ann. of Math. Logic 12 (1977), 59-111.

[PV] H. J. Prömel and B. Voigt, Baire sets of k-parameter words are Ramsey, Trans. Amer. Math. Soc. 291 (1985), 189-201.

[PSV] H. J. Prömel, S. G. Simpson and B. Voigt, A dual form of Erdös-Rado's canonization theorem, J. Combin. Theory Ser. A, 42 (1986), 159-178.

[She] S. Shelah, Can you take Solovay's inaccessible away?, Israel J. Math. 48 (1984), 1-47.

[Sol] R. M. Solovay, A model of set theory in which every set of reals is Lebesgue measurable, Ann. of Math. 92 (1970), 1-56.

Forschungsinstitut für Diskrete Mathematik, Universität BonN, Nassestrasse 2, 5300 Bonn 1, Federal Republic of Germany 